\title{
High-Speed Microcontact Printing
}

\author{
Jo A. Helmuth ${ }^{\ddagger}$, Heinz Schmid ${ }^{\star \dagger}$, Richard Stutz ${ }^{\dagger}$, Andreas Stemmer ${ }^{\ddagger}$, and Heiko Wolf ${ }^{\dagger}$. \\ Nanotechnology Group, ETH Zurich, CH-8092 Zurich, Switzerland, and IBM Research GmbH, Zurich Research \\ Laboratory, 8803 Rüschlikon, Switzerland
}

\section{Experimental Setup:}

A scheme of the mechanical and optical parts of the printing tool is shown in Figure 1. The positioning of stamp and substrate is done with two linear stages (Limes 90, OWIS) driven by stepper motors. The fast short-range movement of the stamp towards the substrate is realized with a feedback controlled piezo actuator (P-841.60, E509.X1, Physik Instrumente). The linear stages and the piezo are controlled with LabVIEW. Stamp and substrate can be aligned with a two-axis manual tilting stage on which the substrate is held tightly in place by a vacuum holder. The stamp is mounted on the piezo via an adapter (stamp holder) that allows illumination and visual examination of the stamp-substrate contact with a CCD camera via a mirror and a beam-splitter.
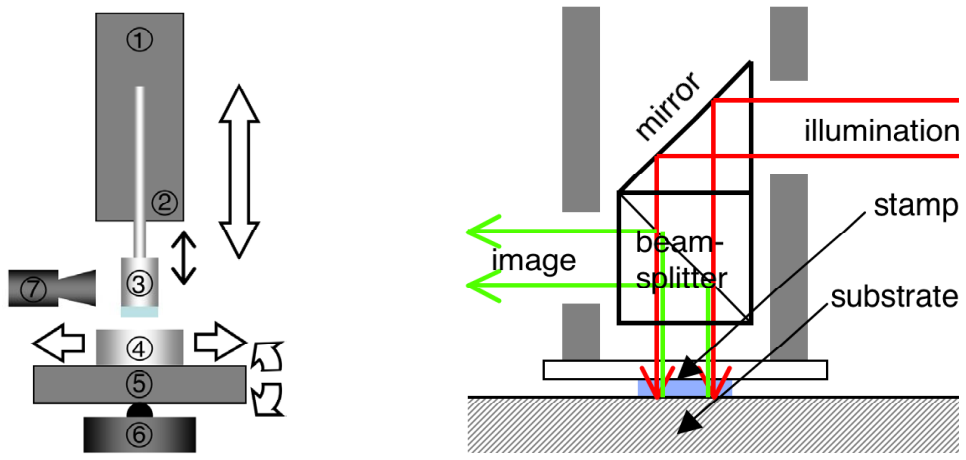

Figure 1: Left) Scheme of the mechanical part of printing tool. The two linear stages (1 and 5), the piezo (2), the stamp holder (3), the substrate holder (4), the tilting stage (6) and the camera (7) are shown. Right) Schematic cross-section of the stamp holder.

\section{Stamps and Inkpads:}

Stamps are produced by curing liquid PDMS (Sylgard, Dow Corning) in a cavity between a $175 \mu \mathrm{m}$ thin glass backplane and a master that contains a negative of the desired structures. The masters are Si-wafers with surface structures made by standard photo-lithography and coated with a plasma deposited teflon-like anti-sticking layer. The line edge roughness of the master is given by the photolithography step and is accurately reproduced in the PDMS stamp as evident in Fig 3 for example. The glass backplane it is treated with allyltrichlorosilane (ABCR) vapor prior to cast molding to promote a strong bonding with the PDMS. A thin elastomeric layer allows faster separation from the substrate compared to a thicker PDMS layer but requires better mechanical alignment. We found that a stamp thickness of $100 \mu \mathrm{m}$ is a good compromise. PDMS shrinks during the curing which results in a concave stamp surface that causes trapping of air bubbles. A stepped edge along the periphery of the stamp alleviates this problem. Stepped edges are fabricated by an additional $30 \mu \mathrm{m}$ thick layer of photoresist on the Siwafer surrounding the stamp area. Low molecular weight PDMS impurities are thoroughly extracted from the stamps by rinsing with ethanol. Finally, the stamps are glued on $1 \mathrm{~mm}$ thick glass slides for ease of handling.

Inkpads were made by curing a 3-mm-thick PDMS layer in polystyrol petri-dishes. Prior to exposure to the ink, the inkpads are washed in a large volume of ethanol for $48 \mathrm{~h}$ to extract uncured PDMS. Solved ethanol can evaporate from the inkpads within $48 \mathrm{~h}$. A drop of liquid hexadecanethiol is pipetted onto one inkpad and by placing a second inkpad of equal size on the drop, distributed over the two inner PDMS surfaces. The exact amount of ink supplied is measured gravimetrically with a high-precision balance. The inaccuracy of the balance is on the order of micrograms and thus negligible. After about $48 \mathrm{~h}$, the ink concentration in the inkpads can be assumed to be uniform. Stamps are inked by pressing them onto the former inner side of an inkpad. After $24 \mathrm{~h}$,

\footnotetext{
$\ddagger$ ETH Zurich

Zurich Research Laboratory
} 
the stamp has adopted the ink concentration from the inkpad. The volume of the stamp is negligible compared with that of the inkpad, thus the concentration in the pad is practically unchanged by the inking of one stamp.

\section{Numerical Diffusion Simulation:}

The equation for one-dimensional diffusive mass transport is numerically integrated by applying a finite difference scheme. Inking of stamps is a one-dimensional problem. However, in the case of printing, this simplification is only valid if only the outer regions of the stamp are influenced by the printing process which is a printing condition that this work aims at. The stamp-substrate interface is modeled with a perfect sink boundary condition, assuming that any ink at the interface will instantaneously bind to the substrate. The total flux per area over the interface is monitored and a no-flux boundary condition is enabled once the total flux equals the surface concentration of a monolayer of HDT. Inking is modeled with a step function as initial condition. Effects of the interface between stamp and inkpad on the mass transport are neglected, as both are made from the same type of PDMS. For the back-side of stamps and inkpads no-flux boundary condition are used.

\section{Electrical Evaluation of Contact Dynamics:}

Complementary to the optical contact measurements electrical contact measurements were performed to investigate the characteristics of the setup and the impact dynamics at high print speeds. A steel sphere is used as upper electrode. The contacted surface is either an aluminum plate or a thin layer of PDMS that is made conducting with a layer of gold [1]. All other components of the printing tool and the printing procedure are unchanged. A DC voltage is applied to the sphere and the contacted surface. The voltage drop across a resistor in series to the contact is measured giving an on/off signal corresponding to a contact/no-contact. In figure 2 results are plotted for the sphere hitting a hard respectively a soft surface. For the hard-hard pairing a contact bouncing is observed. The frequency of the oscillation is constant at about $0.8 \mathrm{kHz}$. The impact of the sphere on the surface induces vibrations that make the sphere bounce off the surface a few times until the vibrational energy has been dissipated. If the same sphere is printed on a soft slab of a Au coated PDMS the electrical contact is immediately formed and stable due to the compliance and efficient damping of the oscillation in PDMS. (The Au coating reduces the high surface free energy of native PDMS; surface adhesion forces are not expected to play an important role) We conclude that an electro-mechanical switch where at least one contact is made from an elastomer can operate at high speeds while having electro-mechanically well defined on-off contact states. Artifacts in micro-contact printed patterns due to contact bouncing are not expected in our high-speed printing setup.
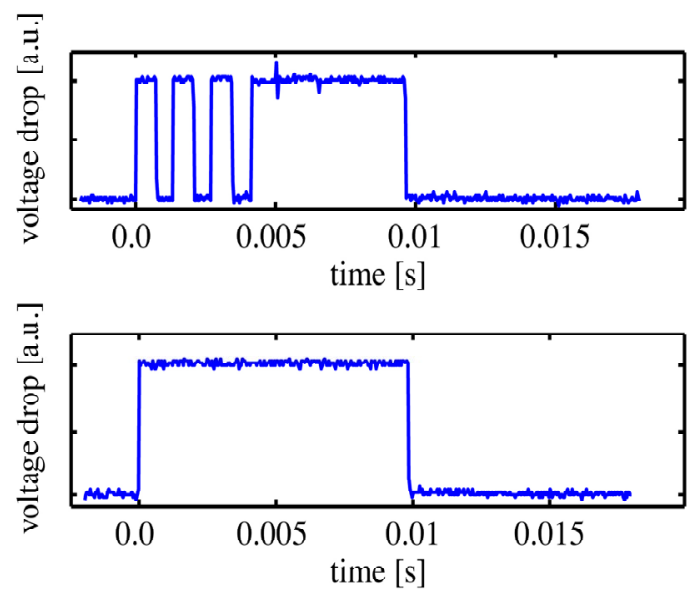

Figure 2: Electrical contact dynamics measurements. Top) Hard-hard pairing. The voltage drop oscillates indicating that the contact is made and lost several times. Bottom) Hard-soft pairing. No oscillations can be observed. The net contact time equals the programmed contact time of $10 \mathrm{~ms}$. 
Table of the experimental results of printed and etched Au films:

\begin{tabular}{|l|l|l|l|l|}
\hline & $\mathrm{C}=1.9 \mathrm{mM}$ & $\mathrm{C}=10.8 \mathrm{mM}$ & $\mathrm{C}=16.6 \mathrm{mM}$ & $\mathrm{C}=35.1 \mathrm{mM}$ \\
\hline $\mathrm{t}=1 \mathrm{~ms}$ & $\mathrm{c}$, very poor & $\mathrm{c}$, poor & c, good & c, good \\
\hline $\mathrm{t}=2 \mathrm{~ms}$ & & & & very good \\
\hline $\mathrm{t}=3 \mathrm{~ms}$ & & & very good & \\
\hline $\mathrm{t}=4 \mathrm{~ms}$ & & $\mathrm{c}$, good & & $\mathrm{s}$, fair \\
\hline $\mathrm{t}=5 \mathrm{~ms}$ & $\mathrm{c}$, very poor & & $\mathrm{s}^{*}$, good & \\
\hline $\mathrm{t}=8 \mathrm{~ms}$ & & $\mathrm{~s}^{*}$, good & & \\
\hline $\mathrm{t}=10 \mathrm{~ms}$ & & & $\mathrm{~s}$, fair & $\mathrm{s}$, poor \\
\hline $\mathrm{t}=15 \mathrm{~ms}$ & $\mathrm{c}$, very poor & $\mathrm{s}^{*}$, good & & \\
\hline $\mathrm{t}=30 \mathrm{~ms}$ & & $\mathrm{~s}$, poor & $\mathrm{s}$, poor & $\mathrm{s}, \mathrm{v}$, very poor \\
\hline $\mathrm{t}=35 \mathrm{~ms}$ & $\mathrm{c}$, very poor & & & \\
\hline $\mathrm{t}=100 \mathrm{~ms}$ & $\mathrm{c}, \mathrm{s} *$, very poor & $\mathrm{s}$, poor & $\mathrm{s}, \mathrm{v}$, poor & $\mathrm{s}, \mathrm{v}$, very poor \\
\hline $\mathrm{t}=1 \mathrm{~s}$ & $\mathrm{c}, \mathrm{s}, \mathrm{v}$, very poor & & & \\
\hline
\end{tabular}

Table 1: Quality of printed and etched samples as function of the ink concentration and print time. The first letter indicates the type of degradation, followed by the resulting quality of the pattern. c: incomplete SAM, v: vapor phase diffusion, s: surface diffusion more that $50 \mathrm{~nm}$, and $\mathrm{s}^{*}$ : surface diffusion below $50 \mathrm{~nm}$.

[1] Schmid, H.; Wolf, H.; Allenspach, R.; Riel, H.; Karg, S.; Michel, B.; Delamarche, E. Adv. Funct. Mater. 2003, 13, 145153 